\title{
Les consignes en classe de langue : activité polyfocalisée et rôle du regard
}

Quel apport des discours réflexifs et quels enjeux de formation?

\section{Estefania Dominguez et Véronique Rivière}

\section{OpenEdition}

\section{Journals}

Édition électronique

URL : http://journals.openedition.org/rdlc/715

DOI : $10.4000 /$ rdlc. 715

ISSN : 1958-5772

Éditeur

ACEDLE

Référence électronique

Estefania Dominguez et Véronique Rivière, «Les consignes en classe de langue : activité polyfocalisée et rôle du regard ", Recherches en didactique des langues et des cultures [En ligne], 12-2 | 2015, mis en ligne le 20 novembre 2015, consulté le 19 avril 2019. URL : http://journals.openedition.org/rdlc/715 ; DOI : $10.4000 /$ rdlc. 715

Ce document a été généré automatiquement le 19 avril 2019.

\section{(i) $\$$

Recherches en didactique des langues et des cultures is licensed under a Creative Commons AttributionNonCommercial-NoDerivatives 4.0 International License 


\section{Les consignes en classe de langue : activité polyfocalisée et rôle du regard}

Quel apport des discours réflexifs et quels enjeux de formation?

\section{Estefania Dominguez et Véronique Rivière}

Tous nos remerciements vont à Sher Bennet, enseignante de français à Vancouver, pour la traduction.

\section{Introduction}

1 L'activité de consigne est une activité majeure du processus d'enseignement et d'apprentissage. Ses enjeux sont bien connus : clarté informationnelle, compréhension des attentes de l'enseignant, autonomie, etc. Elle se définit comme :

l'exercice d'une contrainte, plus ou moins directe et plus ou moins forte, sur l'activité de l'apprenant en vue d'améliorer ses compétences langagières. Elle ressortit de la fonction d'animation et d'information de l'enseignant [...] et est l'écho d'un projet porté par celui-ci et/ou par l'institution éducative (Rivière, 2006 :

19).

2 De ce fait, l'activité prescriptive constitue un puissant levier de la réalisation du projet de l'enseignant et de la mise au travail des élèves. Elle est aussi, d'un point de vue théorique, consubstantielle à l'action dans la mesure où ces discours ont une forte dimension illocutoire et un impact sur l'agir apprenant.

3 Néanmoins, la dimension cachée de l'activité de consigne (les préoccupations de l'enseignant, les normes professionnelles, les intentions, les motifs présidant à l'élaboration et la distribution des consignes, les micro-décisions) demeure encore peu renseignée (voir toutefois Dominguez, 2013). C'est, par conséquent, dans cette perspective que nous abordons l'activité de prescription.

4 Après avoir défini le contexte d'étude et rappelé certains principes théoriques et méthodologiques inhérents aux études sur l'agir enseignant s'appuyant sur des discours 
réflexifs, nous nous intéresserons à la dimension polyfocalisée de l'activité de consigne et de mise au travail des apprenants. En effet, l'activité enseignante requiert constamment une multi-activité (Rivière \& Bouchard, 2012) qui nécessite des "arts de faire" et qui revêt une importance particulière durant la passation des consignes du fait de l'enjeu de mise en activité des élèves et de l'enjeu d'actualisation du projet de l'enseignant dans le vif de l'action.

5 Nous mettrons l'accent, dans une troisième partie, sur une modalité de gestion de la multi-activité : l'activité visuelle de l'enseignant associée à la passation des consignes. En effet, celle-ci (mais on peut sans doute généraliser à l'ensemble de l'activité de l'enseignant) requiert une activité d'observation intense et le regard constitue un mode de lecture normé des événements sur lesquels il doit réagir in situ. Mais l'enseignant doit aussi organiser l'activité attentionnelle des élèves, organiser visuellement et sémiotiquement la transposition des savoirs langagiers à acquérir (à travers les supports pédagogiques et autres artefacts). Tout ceci engage des "méthodes" contribuant à définir et comprendre l'action enseignante.

Cela nous conduira finalement à mettre en rapport la situation alliant visionnage de l'action et discours réflexif avec la situation de classe où l'enseignant est aussi un observateur au regard aiguisé. Nous esquisserons ainsi les contours d'une "vision professionnelle" (Goodwin, 1994) partagée et intéressante à mobiliser au regard des enjeux de formation des enseignants.

\section{Éléments de contextualisation}

\section{Présentation du corpus}

7 Notre réflexion s'appuie sur deux corpus de discours réflexifs. Nous les présentons dans cette section et en discutons les présupposés méthodologiques dans la section suivante. Le premier corpus, constitué en 2012 concerne trois enseignantes de FLE expérimentées (Victoria, Isabelle et Cécile) qui exercent en centre de langues universitaire en France. Les cours dispensés s'adressent à un public adulte international de niveau B2.

La vidéo étant un moyen d'accéder au réel, nous avons tout d'abord filmé quatre séances de cours (d'une heure chacune) afin de recueillir les pratiques effectives des enseignantes. L'activité verbale, gestuelle, matérielle, corporelle a été retranscrite. La présentation du projet aux informatrices n'a été présenté que dans les grandes lignes (pas de définition précise du sujet: l'activité prescriptive). Puis, les informatrices ont été sollicitées un mois après le filmage pour réaliser des entretiens d'auto-confrontation. Ceux-ci se sont déroulés sur le lieu de travail des informatrices. Dans ce contexte professionnel, le chercheur présente des fragments de cours vidéoscopés et les enseignantes sont amenées à les commenter. Il faut préciser que, d'une part, ces fragments ont été choisis en amont par le chercheur dans le but de proposer uniquement les passages prescriptifs; et que d'autre part, nous avons aussi décidé de ne pas dévoiler le sujet afin de laisser advenir la mise en discours de manière plus libre. Pour ce faire, il leur était demandé de parler de l'action visionnée et les relances ne faisaient que réorienter leur discours dans ce but. Enfin, les entretiens d'auto-confrontation ont également été transcrits. 
9 Le second corpus a été constitué en 2004 dans le cadre d'un projet de recherche sur la construction du "répertoire didactique" de l'enseignant de langue. Il concerne une étudiante de Master, Marie-Laure, enseignant pour la première fois en centre universitaire face à des étudiants de niveau B2. Dans cette étude, les cours ont été enregistrés sur support audio, mais n'ont pas donné lieu à auto-confrontation. Les discours réflexifs consistent plutôt en débriefings, dirigés par une conseillère pédagogique, tout de suite après la tenue du cours. Ils se déroulent donc dans le cadre de la formation à l'enseignement, contrairement au premier corpus qui a été constitué dans une perspective de recherche.

\section{Statuts des discours réflexifs et rationalité de l'action} enseignant. Premièrement, durant l'auto-confrontation, l'enseignant sort de son cadre habituel et change de statut. "D'acteur sur le terrain, tenant un rôle prédéterminé auprès de ses élèves, il devient un observateur de lui-même, sollicité pour produire de la signification à propos de ses actions", il devient alors un "chercheur dans le contexte" (Cicurel, 2011 : 250-251). Deuxièmement, l'enseignant a "tendance à adopter une position d'expertise ${ }^{1}$ par laquelle il désire établir la professionnalisation de son agir" (Ibid. : 252), ce qui peut l'amener à des généralisations et à des restrictions dans l'accès aux détails de l'action étudiée. Aussi, les verbalisations ne sont pas les pratiques effectives car il peut exister une "disparité entre ce que [l'enseignant] fait et ce qu'il dit qu'il a fait" (Ibid. : 255). On ne saurait considérer ces discours comme "la" pensée de l'enseignant, fidèle à la pensée in vivo dans le cours de l'action. Il s'agit d'une pensée reconstruite et présentée au chercheur dans la situation d'entretien. Le chercheur doit composer avec ces paramètres.

\section{Agir prescriptif et activité polyfocalisée}

13 L'activité didactique s'appuie sur une intrication étroite et simultanée entre activité verbale, interactionnelle et activité matérielle. L'enseignant, comme les apprenants, sont constamment engagés dans des foyers d'attention et d'activités multiples, concomitants ou concordants. Par exemple, lors de la correction collective d'un exercice, les apprenants dirigent leur attention vers les réponses fournies par le groupe ou par un apprenant en particulier, et simultanément s'attachent à corriger, rectifier, annoter des éléments sur leur cahier, en regardant les notes au tableau, et à comprendre leurs erreurs. En bref, l'activité cognitive et physique est doublement orientée. L'apprenant est alors engagé dans un double foyer d'attention. Pour peu que son camarade le sollicite sur un point précis et momentané, le voilà dans une triple focalisation au moins, l'une individuelle

Recherches en didactique des langues et des cultures, 12-2 | 2015 
(celle de noter la correction et de prêter attention à son exercice), les autres conjointes, mais toutes orientées distinctement. De son côté, l'enseignant est occupé à fournir des corrections plus ou moins exhaustives, contextualisées, exemplifiées, etc., et en même temps à répondre aux sollicitations, questions, remarques des apprenants, à organiser aussi l'exposition au savoir (que note-t-il au tableau? met-il de la couleur? comment hiérarchise-t-il les éléments de correction? etc.). Ces mouvements de cadre se réalisent en fonction des (micro)finalités qui animent les interactants. À partir des travaux de Goffman, Filliettaz (2002: 98) a théorisé, dans le cadre d'une théorie de l'action, cette polyfocalisation, de nature configurationnelle ou individuelle. Cette dernière, plus adaptée à notre objet, l'agir enseignant, est définie comme :

des situations dans lesquelles les agents manifestent simultanément un engagement praxéologique dans deux directions différentes, orientées vers des foyers distincts. Ces engagements peuvent porter tous deux sur des finalités de nature conjointe, mais plus fréquemment, ils coordonnent une action conjointe et une action individuelle (ibid. : 99).

Ce que nous souhaitons mettre en évidence ici est bien le sens que recouvre, pour l'enseignant, son action individuelle de prescription, enchâssée dans une action conjointe. Il nous semble que cette polyfocalisation, imbriquant actions et discours, repérable dans les séquences de consignes, contribue à instaurer le cadre didactique et pédagogique de travail commun. Il constitue, par conséquent, une pierre de touche à l'efficacité de leur transmission et induit des choix à opérer (et un processus de décision à mener). L'enseignant dispose à ce sujet des savoirs d'action de type organisationnel, variables selon l'expérience. Les discours réflexifs produits en auto-confrontation retracent généralement les étapes des prises de décisions et dévoilent les raisons qui les sous-tendent. Ce qu'il convient alors d'observer, c'est ce que dit l'enseignant au sujet de cette polyfocalisation de l'attention, la sienne propre et surtout celle des apprenants.

\section{Une gestion délicate des focalisations}

Cette intrication des actions et des discours n'est pas toujours facilement gérable dans l'interaction didactique. Elle s'avère même parfois problématique pour des enseignants qui débutent dans la profession. Il en va ainsi de Marie-Laure, enseignante novice, pendant son stage de maitrise FLE à l'université Paris 3, s'adressant à des étudiants de niveau intermédiaire ${ }^{2}$. Dans l'extrait ci-dessous, elle entame une activité didactique d'expression orale à partir de publicités de la presse écrite. Son objectif est d'amener les étudiants à dresser "les caractéristiques du fonctionnement d'une publicité" et d'étudier "les jeux sur le sens propre et le sens figuré". Elle offre un guidage de l'activité à travers quatre tâches: "décrire l'image rapidement, voir la place du produit dans l'image, étudier le rapport entre l'écrit et l'image, dire qui est visé et pourquoi". À ce moment de l'interaction, elle a déjà distribué les supports publicitaires (papier) et distribue les dernières consignes ${ }^{3}$. 


\begin{tabular}{|c|c|c|}
\hline 27 & ML & $\begin{array}{l}\text { alors + vous vous regrouperez les personnes qui ont la même publicité + vous vous } \\
\text { regrouperez avec qui vous mettrez une petite chaise devant + puisque vous allez } \\
\text { avoir : : je pense euh : : dix minutes + pour travailler en groupe + X les publicités X } \\
\text { discuter + je vais vous marquer au tableau les questions + ce que vous avez regardé } \\
\text { particulièrement + dans la publicité + + alors d'abord + vous allez décrire rapidement } \\
+ \text { l'image + + + (elle écrit au tableau }) \mathrm{X}+++ \text { ça au tableau (elle écrit au tableau) alors si je } \\
\text { vous dis + vous allez regarder la place du produit dans la publicité + X ça veut dire } \\
\text { quoi +++ (elle écrit au tableau) la place du produit dans la marge + le produit ça veut } \\
\text { dire quoi ?++ X répondez pas + j'ai pas l'impression de parler toute seule + X c'est } \\
\text { formidable ++ alors + donc je X + la place du produit + dans l'image }\end{array}$ \\
\hline 28 & As & (discussions en groupe) \\
\hline 29 & ML & $\begin{array}{l}\text { là c'est suffisamment un vide }+ \text { faire un vide }+\mathrm{X}+++ \text { un vide } \mathrm{X}++\mathrm{j} \text { 'essaie de } \\
\text { communiquer avec vous }+\mathrm{X} \text { tableau }\end{array}$ \\
\hline 30 & As & (rires) \\
\hline 31 & ML & j'essaie de communiquer avec vous $\mathrm{X}+$ excusez-moi (rires) \\
\hline 32 & As & (rires) \\
\hline 33 & ML & $\begin{array}{l}\text { vous êtes dans un petit groupe }+ \text { vous allez réfléchir sur la publicité }+ \text { mais vous allez } \\
\text { réfléchir en répondant aux quatre questions }++ \text { en suivant les quatre points de } \\
\text { réflexion que je vous propose ici + donc je X au tableau + mais avant de vous lancer } \\
\text { dans la discussion }+ \text { vous X des connaissances }+ \text { et je voudrais vérifier si vous } \\
\text { compreniez les mots }+ \text { donc quand je vous dis la place du produit }+X \text { voilà }\end{array}$ \\
\hline 34 & As & (rires) \\
\hline 35 & ML & (elle continue à écrire les questions au tableau) \\
\hline 36 & Am & $\mathrm{x}$ \\
\hline 37 & ML & oui $?$ \\
\hline 38 & Am & $\mathrm{x}$ \\
\hline 39 & ML & euh : s'il y en a oui \\
\hline 40 & Am & $\mathrm{X}$ produit \\
\hline 41 & ML & mais alors le produit c'est quoi ça le produit là \\
\hline 42 & Am & $\mathrm{X}$ \\
\hline 43 & ML & $\begin{array}{l}\text { d'accord + voilà + X à préciser ++ + (elle continue à écrire les questions au tableau) comme } \\
\text { je dis + entre le rapport entre l'écrit + et l'image + est-ce que vous avez une idée de : : ce } \\
\text { que je veux dire par là ?+++ (elle continue à écrire les questions au tableau) oui ? }\end{array}$ \\
\hline
\end{tabular}


Cet extrait met en lumière, du fait des problèmes de communication que Marie-Laure rencontre avec les apprenants au début de cette séquence, (27: "j'ai pas l'impression de parler toute seule" - 31: "j'essaie de communiquer avec vous"), plusieurs difficultés dans la gestion ordonnée des actions et des discours, dans la mise en œuvre de son action :

- difficulté pour gérer l'activité de discours :

- de consigne quand il lui faut prescrire aux apprenants, selon un certain ordre, des actions concrètes (se mettre en groupe, faire un groupe par publicité, discuter, réfléchir) et des actions langagières spécifiques ("décrire l'image", "regarder la place du produit", "répondre aux questions", "faire l'interprétation de l'image") et

- de sollicitation parce que son but est aussi de vérifier la compréhension des consignes ( 27 : "quelle est la place du produit" ; 41 : "le produit ça veut dire quoi") et/ou d'expliciter ses attentes ( $43:$ "est-ce que vous avez une idée de ce que je veux dire");

- difficulté pour gérer l'organisation des actions de discours énumérées ci-dessus et de ses propres actions concrètes (écrire au tableau les consignes en regardant ses notes), qui l'obligent à se détourner physiquement.

De plus, elle doit à un certain moment retrouver le bon canal de communication, brouillé parce que les apprenants, de leur côté, sont focalisés sur l'observation et le commentaire apparemment amusant (rires) et hors cadre des publicités. Marie-Laure est orientée simultanément vers un enjeu communicationnel d'intercompréhension et vers un double enjeu praxéologique dirigé vers l'activité collective (faire démarrer une activité de groupe) et vers son activité propre (écrire au tableau les questions). La stagiaire exprime d'ailleurs, dans les commentaires sur sa propre action, réalisés après la séance de cours, ou dans son bilan personnel écrit, ce triple enjeu difficile à mener conjointement.

CCML5

\begin{tabular}{|c|c|c|}
\hline 010 & ML & $\begin{array}{l}\text { donc euh dans l'ensemble euh bon l'introduction ça allait la mise en place des } \\
\text { groupes donc j'aurais dû d'abord euh préparer mes consignes parce que du } \\
\text { temps que j'écrivais moi j'pensais d'abord les écrire pendant qu'ils s'mettaient } \\
\text { en place XXXX }\end{array}$ \\
\hline 011 & $\mathrm{x}$ & (rire) (intonation théâtrale) coucou c'est moi \\
\hline 012 & ML & $\begin{array}{l}\text { ben oui mais euh : donc là j'ai un peu mal géré mon truc parce que après il fallait } \\
\text { d'abord que je me fasse entendre }\end{array}$ \\
\hline 013 & $\mathrm{x}$ & non mais t'as rattrapé le coup \\
\hline 014 & ML & $\begin{array}{l}\text { en fait j'pensais que gentiment pendant que j'écrivais i's'mettaient en groupes } \\
\text { et qu'après i regarderaient XXX }\end{array}$ \\
\hline 015 & $\begin{array}{l}\mathrm{X} \\
(\text { Stag.) }\end{array}$ & XXX oui Marie-Laure \\
\hline
\end{tabular}

Bilan personnel écrit: "critiques négatives - J'ai mal géré l'annonce des consignes. Au lieu d'écrire au préalable au tableau les quatre points qu'ils devaient étudier, je leur ai d'abord distribué les publicités et je leur ai demandé de se mettre en groupe. C'était donc le bazar et j'ai eu du mal à me faire entendre !" 

l'alternance parfois nécessaire des actions concrètes et des actions de communication. Marie-Laure pensait, avant d'entrer en classe, que certaines actions pouvaient se réaliser en même temps que certains discours (écrire au tableau et vérifier la compréhension des consignes) ou certaines autres actions (écrire au tableau et se mettre en groupe) (cf. les conjonctions ou adverbes de temps ${ }^{4}$ ). Ces commentaires explicitent un point majeur de la distribution des consignes, et plus généralement de la méthodologie d'enseignement, celui d'un ordre temporel des actions et discours, du moment opportun de certaines actions ou discours et du passage délicat entre des engagements ou des focalisations différentes.

\section{Polyfocalisation et prise de décision}

19 Si nous nous penchons maintenant sur les discours réflexifs d'enseignantes plus expérimentées, nous retrouvons des situations similaires de gestion simultanée des actions et des discours et de focalisations multiples durant la passation de consignes. Observons à quelles pensées elle donne lieu.

\section{Réduire ou non les foyers d'attention?}

L'enseignante, Isabelle, lance une activité qui porte sur la correction d'un exercice de définition de différents termes sur l'université. C'est l'étape première qui va permettre de découvrir le système universitaire français, celui-ci étant l'objet de la séance (sous forme de présentation PowerPoint).

Alors, je vous ai préparé un Powerpoint et vous allez trouver la réponse aux différentes questions, d'accord? Est-ce que je vous donne les papiers maintenant ou est-ce que vous écoutez? Alors, avant je voudrais que vous me disiez les réponses que vous avez trouvées. Pour vous c'est quoi l'université ? Qui a trouvé la définition?

Elle verbalise ainsi :

alors LÀ euh : : je je commence plusieurs choses et euh en fait je DÉCIDE sur le moment dans quel ordre je vais faire les choses c'est-à-dire que : au début je voulais lancer le Powerpoint mais en même temps je voulais qui + qu'ils me disent ce sur quoi ils avaient réfléchi par groupes et puis en même temps j'hésitais à leur donner les papiers du power point [...] je me disais s'ils ont les papiers ils ne vont pas regarder enfin bon donc je décide finalement qu'on réponde ensemble aux questions + RAPIDEMENT pour pas passer trop de temps parce que je SAIS qu'on va y revenir après ++++ + donc voilà +++ mais euh je décide sur le moment ça (E-Isa, P23).

Tous les éléments organisationnels énoncés dans la consigne (artefact d'appui Powerpoint, modalités de participation (suivre avec le papier ou écouter), tâche énoncée (donner les réponses) sont repris dans le commentaire d'Isabelle. Tout d'abord, elle y pose d'emblée comme un fait cette attention multiple et simultanée à travers cette assertion " je commence plusieurs choses" et la manière dont elle va la gérer ("je DÉCIDE sur le moment dans quel ordre je vais faire les choses"). Deux actions semblent avoir été prévues en amont (" je voulais et en même temps je voulais") et une ne semble pas l'avoir été ("j'hésitais"). L'ordre précis, dans lequel elle prescrit, n'est pas prévu dans le détail. Cela fait apparaitre une 
micro-décision prise dans le vif de l'action et obéissant à une forme de règle ("je me disais si...") sur les conduites supposées des apprenants. Finalement, ce qui influence son choix est le facteur temps dont elle a la maîtrise ("je SAIS qu'on va y revenir après") et l'objectif d'apprentissage : elle décide de corriger d'abord car elle sait (par anticipation) que celleci prépare l'activité à partir du Powerpoint. Les réponses des apprenants sont un moyen d'évaluer leurs connaissances initiales sur le système universitaire.

Sa décision consiste donc à réduire les foyers d'attention (pas de distribution de polycopié afin qu'ils se focalisent tous sur la réponse aux questions). L'ordre choisi de ces microactions lui permet d'optimiser le processus d'apprentissage. Les savoirs de l'enseignante ici identifiables sont :

- sa capacité à sérier une (micro-)action dans le moment présent et à jauger sa pertinence au regard d'une programmation plus globale,

- sa capacité à anticiper les effets de son action sur la gestion des foyers d'attention au sein de la classe.

\section{Prendre une respiration}

Ce qui pourrait caractériser une gestion fluide de la simultanéité des actions, des discours et des foyers d'attention durant la passation des consignes, c'est la capacité à s'extraire momentanément du cours de l'action, de l'évaluer, puis de l'ajuster et la compléter, en fonction de la lecture que fait l'enseignante de la situation présente au regard de sa planification. Ainsi, dans cet extrait :

Alors, on va continuer, on va aujourd'hui observer les pages de garde, d'accord. Seulement la première page. Donc, la semaine dernière, vous avez étudié les différentes parties qui constituent un dossier, un mémoire et un rapport de stage et aujourd'hui, on va se focaliser sur la première page [...] alors vous allez travailler tous les trois [...].

L'enseignante Victoria énonce une consigne qui demande une gestion de la disposition des apprenants (ils doivent travailler en groupes de trois), la gestion de documents utiles à l'activité (des exemples de mémoire, de rapports de stage et de dossiers) et la gestion de la communication (dire ce qu'ils vont faire). Pendant cette énonciation, elle se déplace et distribue à chaque groupe un support (mémoire, rapport de stage ou dossier). Lorsque cette activité, qui l'engage verbalement et physiquement, se termine, elle vérifie le bon agencement de tous les paramètres utiles à son action et a la possibilité de prendre du recul. Trois secondes plus tard, elle ajoute :

Alors, je vous dis la consigne quand même parce que là, vous avez seulement le dossier à observer. Vous avez un document à compléter, comme la semaine dernière. Vous observez ce que vous trouvez sur la première page des dossiers, rapports et mémoire.

Lors de la verbalisation, Victoria donne à voir la dynamique réflexive qui conduit à l'ajout :

Alors là je me rends compte que euh enfin je me rends compte +++ oui +++ oui je me rends compte que euh je les ai mis en petits groupes mais ils ont les dossiers donc ils ont les supports à observer mais ils n'ont pas la fiche euh la fiche euh pédagogique qui accompagne et qui euh qui donc euh qui COMPORTE l'activité que je leur demande DONC je : : : : rectifie j'ai envie de 
dire le tir je sais pas s'il y a un ordre à à : : : : à respecter d'abord la fiche puis les placer mais en tout cas là c'est comme ça que j'ai fait et : du coup je rectifie en en les en leur donnant le document et là je passe de groupe en groupe pour donner la consigne euh: (souffle) je sais pas si c'est:: : (hésitante) terrible euh on a plutôt tendance à donner les consignes de manière collective euh : là je : + je je vais dans chaque groupe et et : et je réexplique à chacun euh +++ je SAIS que euh ils sont plus concentrés quand je vais les voir individuellement enfin quand je vais le voir en sous-groupe ils m'ÉCOUTENT davantage que quand je suis en : : devant la classe entière +++++ ils doivent se sentir plus concernés $\uparrow$ je sais pas $\downarrow$ (E-Vic,P5) durant ce moment où elle s'est tue ("je me rends compte que euh enfin je me rends compte +++ ouit++ je me rends compte") et donne à voir comment elle diagnostique la situation (ce que les apprenants ont et n'ont pas pour réaliser l'activité). Cela la conduit à "rectifier le tir" et par conséquent à modifier la trajectoire de la prescription prévue. Cette énonciation en deux temps (non prévue initialement) a des conséquences sur la gestion du cadre participatif. Alors que la première phase de la consigne s'est gérée à la cantonade, la deuxième phase nécessite une gestion plus individualisée, par groupes. Cette différence tient au fait que les apprenants sont déjà engagés et qu'elle ne souhaite pas défaire la focalisation déjà amorcée sur l'activité. Et face au chercheur, elle envisage cette façon de faire comme potentiellement non conforme à un idéal-type ("je sais pas s'il y a un ordre à respecter mais en tout cas c'est comme ça que j'ai fait- on a plutôt tendance à donner les consignes de manière collective"), mais qui présente des avantages ("ils m'ÉCOUTENT davantage que quand je suis en :: devant la classe entière") au regard de sa connaissance des publics ("je SAIS qu'ils sont plus concentrés").

Finalement, l'enseignant, pris dans la dynamique communicationnelle, est engagé en tous sens : la parole, le regard, les gestes, la gestion des artefacts, etc., Quand un moment de silence advient, que son activité attentionnelle est allégée, il peut passer en revue son action, son discours et l'action des apprenants et identifier le repérage d'un oubli par exemple. En somme, il met en relation, par un processus de traitement de l'information, l'état de la mise au travail et son projet. S'extraire un instant de la communication orale facilite l'activité réflexive et analytique de la situation et fait partie de l'activité prescriptive. Cette respiration, cette nécessité de réfléchir "hors du feu de l'action" (Perrenoud, 2001) est une composante de l'agir prescriptif.

Goffman appelait de ses vœux à envisager "la totalité de l'activité dans la situation" (1987 : 153). Les études interactionnelles, notamment dans leur orientation actionnelle, nous permettent de mettre en évidence les configurations multiples des actions et des discours. Les discours réflexifs professionnels permettent, eux, de focaliser sur un autre aspect de cette totalité : le processus de décision, le processus cognitif d'ajustement de l'activité et les formes d'écart par rapport à des représentations normées (et mises en discours) de l'activité enseignante.

\section{Rôle de l'activité perceptive visuelle dans la fabrique de l'action enseignante}

Le moment de distribuer des consignes en vue de réaliser une tâche est rendu délicat par le fait que, premièrement, celles-ci représentent la mise en contact des apprenants, avec 
la langue, objet de savoir, et que deuxièmement l'enseignant y transmet, selon une certaine manière, des informations essentielles et pertinentes à sa réalisation. En particulier, il doit mettre en évidence, rendre visible le projet d'apprentissage, les tâches et les procédures qui y mène et peut-être plus indirectement les savoirs ou savoir-faire à acquérir. Les apprenants, de leur côté, ont à traiter ces informations et, assez souvent, à montrer ce processus de traitement pour (r)assurer l'enseignant qu'ils entrent effectivement dans la tâche d'apprentissage et qu'ils en saisissent les enjeux. Dans les entretiens, les enseignantes très sensibles à ces questions commentent largement les modes de transmission de ces informations et nous remarquons que l'action enseignante de distribution des consignes est notablement soutenue par le souci de rendre évidente et visible les tâches et le guidage de leur réalisation. Les enseignantes ont particulièrement à cœur de soigner cette entrée en matière, de communiquer le mieux possible, ce qui semble nécessiter une mobilisation importante de toutes les ressources. Parmi celles-ci, la modalité visuelle tient une place importante, qu'il s'agisse de la préparation en amont des supports visuels de communication ou qu'il s'agisse d'exercer soi-même une activité perceptive visuelle sur la mise au travail des apprenants.

\section{Faire voir le savoir ou l'activité attendue : favoriser l'accès aux sens}

31 Une première manière de rendre visible un objet est de capter et de guider l'attention de l'interactant sur lui. C'est, en contexte d'enseignement, le rôle des consignes et plus globalement des supports pédagogiques. La présentation de celles-là ou la préparation de ceux-ci consiste notamment à faciliter le repérage, la localisation des informations. Ainsi, au sujet d'une activité menée au laboratoire de langues, Cécile commente :

à gauche du tableau ils savent je mets toujours euh soit les consignes parce que j'ai pas donné un formulaire enfin une fiche pdf ou en général j'essaye de le faire quand même je trouve ça quand même plus + pour EUX je trouve ça plus agréable d'avoir un un VRAI visuel bien fait en plus euh j'essaye de m'appliquer à peu près donc au tableau je mets pour euh pour PAS qui soient perdus je mets OU ils vont trouver les documents donc c'est vraiment euh de la localisation c'est-à-dire que + eux $\uparrow$ ils vont s'y retrouver SURTOUT ceux qui arrivent en retard $\downarrow$ et il y en a toujours à cette heure-là + (P3)

Les objets d'apprentissage sont fortement dépendants de la matérialité didactique, d'un dispositif visuel de guidage et de référenciation et d'un souci de rendre aisé pour l'apprenant l'accès pratique à l'information, ainsi que du souci de produire une réponse à des aléas devenus routiniers (retards). L'enseignante guide l'œil et l'activité cognitive de l'apprenant dans l'espace sémiotique des supports didactiques. De plus, la description de son action permet d'observer la hiérarchisation qu'elle opère entre les supports et leur efficacité visuelle et cognitive : présentement, elle a écrit au tableau les consignes, mais le document pdf est préférable ("quand même je trouve ça quand même plus pour eux je trouve ça plus agréable d'avoir un VRAI visuel"). Le support le plus efficace reste, pour elle, un document écrit et mis en forme dont peut disposer chaque apprenant individuellement.

La dimension scripto-visuelle ou complètement visuelle de son activité ou des ressources utilisées obéit à des stratégies d'enseignement d'accès au sens linguistique ("je note au tableau pour qu'ils le visualisent et qu'ils comprennent bien les MOTS" E-Isa, P19), à des stratégies d'étayage ("c'est visuel on voit que je montre sur le document euh en en PLUS je l'ai présenté de manière à ce que ce soit de manière assez visuelle j'ai groupé dans des rectangles les 
différentes euh PARTIES que je viens de mentionner et ils doivent intituler chaque rectangle voilà "), de différenciation pédagogique entre les apprenants, selon leur progression ou leur profil : "je pense notamment qu'il y en a un le garçon-là qui est au fond qui doit moins enfin comprendre moins rapidement à mon avis il ne peut pas m'apporter la réponse en même temps que les autres à ce moment-là mais j'écris au tableau histoire qu'il voit de quoi je parle" (E-Vic, P12), de redondance informationnelle dans le but de maximiser la transmission ("je les renvoie à une production orale du chapitre CINQ de Édito euh ben comme ça visuellement ils voient la question plutôt que de seulement l'entendre." E-Céc, P8), ou de mise en place d'un format de participation propre à l'activité ("le rapporteur je l'oblige un petit peu à le mettre pour qu'on visualise bien que c'est lui le rapporteur et le meneur de débat" E-Céc, P11). La source visuelle couplée à la source sonore et verbale de la consigne formulée permet d'enrichir l'information donnée. On croise ou on multiplie les modes de réception. Il y a là une manière de mobiliser tels ou tels canaux de communication, de les rendre redondant à dessein (Rivière, 2006).

De même, le recours à certains objets, comme l'utilisation de chapeaux de différentes couleurs correspondant à des rôles dans la mise en place d'un débat, réfère, dans son discours verbalisé, à l'utilisation de signes qui motivent (au sens saussurien du terme d'un ensemble de signes motivés ${ }^{5}$ ) ou renforcent l'apprentissage : "donc j'aime bien c'est pour ça euh que : je fais ce débat avec les chapeaux + c'est parce que je trouve que ça leur permet de + de visualiser leur apport" (E-Céc, P11).

L'enseignant tente également de faciliter l'accès à la finalité des activités qu'il propose. Il éprouve le besoin de rendre visible son action, ses objectifs, et donc de la légitimer :

de plus en plus ouais non j'ai l'impression de le faire de plus en plus d'expliquer euh de VOIR un peu plus loin que le cours en fait ou même DANS le cours quand c'est un cours de 3heures de donner un peu la vision globale (...) Oh c'est en parlant en équipe quoi en disant que c'était intéressant de NOTER ou de dire les objectifs du cours et de d'avoir une vision un peu ça fait partie aussi de du peut-être pour motiver la classe le groupe (E-Isa, P31).

Finalement, l'enseignant est un manipulateur de signes : il les sélectionne, les met en relation, les organise spatialement pour faciliter l'accès au sens linguistique (la langue), praxéologique (la tâche) ou téléologique (la finalité). Il sémiotise de manière continue pour retenir l'attention, maximiser le processus d'apprentissage en développant des pratiques de mise en intelligibilité, de représentation et d'organisation du savoir particulièrement mobilisées dans les moments de distribution de consignes et dont les trois enseignantes ici interrogées semble avoir particulièrement conscience. Et, par son activité corporelle, l'enseignante devient elle-même signe, quand le contexte le requiert :

c'est ce que je fais pour un niveau avancé ce n'est pas ce que je fais pour un niveau débutant en termes de consigne même en termes de communication avec eux par exemple avec un groupe A1 je ne vais pas parler euh quand je suis face au tableau je vais me retourner pour qu'ils me voient pour que ils lisent sur les lèvres un petit peu comme des malentendants quoi c'est utiliser tous les supports possibles euh à la fois gestes euh le le visage et puis le le discours oral pour comprendre" (E-Vic, P34)

Elle semble pousser jusqu'au bout l'articulation entre activité perceptive et cognitive. Cette manière de faire, visant à créer de l'intelligibilité, à rendre plus accessible la tâche, pour les apprenants, est conditionnée par des principes ou une théorie de l'apprentissage 
repérable grâce aux motifs énoncés, forgée au fil du temps et des expériences ("j'ai l'impression de faire de plus en plus") et susceptible d'évoluer ("c'est en parlant en équipe").

\section{L'action visuelle, voir et être vu(e)}

L'enseignant s'applique, en amont ou pendant la classe à rendre optimales les conditions et ressources d'apprentissage en faisant voir du mieux possible aux apprenants, grâce à des supports scripto-visuels travaillés, le projet d'enseignement. Mais son activité consiste également à voir et observer l'activité de l'apprenant. En effet, son activité de jugement et de prise de décision est largement fondée sur son activité perceptive visuelle et sur la prise d'indices pertinents par rapport à ce qui se passe in situ. Le retrait de la scène verbale qu'il opère immédiatement après la distribution d'une première consigne (cf. supra 2.2.2) laisse la place à une activité visuelle intense. À cet égard, l'enseignant pourrait être qualifié de professionnel de la prise de vue, dans la lignée des travaux goodwinien sur la cognition au travail et les "practices of seeing" (Goodwin, 2001). Nous y reviendrons ci-dessous.

Les spécialistes du regard dans l'interaction humaine, comme Kendon (1967), établissent trois fonctions primordiales du regard dans l'interaction: fonction d'expression, de régulation et de contrôle. C'est principalement la troisième fonction dont les enseignantes font état dans les verbalisations. Par exemple, lorsqu'elle énonce, "je suis en train d'aller voir si l'étudiant a bien reçu le ficher", "je vais faire le tour pour voir s'ils peuvent l'ouvrir" (E-Céc, P1), l'activité visuelle permet de compenser une distance dans l'espace (entre l'enseignante et les apprenants installés aux postes informatiques au laboratoire de langues) impliquant un déplacement, mais également une distance entre ses supports d'enseignement et l'apprenant puisque celui-ci est installé à un poste informatique où il est censé recevoir un fichier de travail. L'enseignante n'est plus le seul médiateur puisque la technologie est introduite ; ce qui la pousse à mener une action visuelle et corporelle de vérification de la compréhension de la consigne qui vient d'être énoncée.

OUI ça va être toujours mon fonctionnement en fait euh c'est un peu l'organisation du cours, la consigne, la reformulation, et derrière l'explication si nécessaire ou la vérification la vérification de toute façon au niveau du visuel je vérifie toujours que les étudiants ont bien compris la consigne il y a un des coups de tête hein ça suffit ou des yeux qui partent + des yeux avec des froncements de sourcils pour moi ça me suffit je je du coup je reformule donc je le fais : : j'ai un regard sur les étudiants je PORTE un regard je pense sur les étudiants pour euh parce que j'ai besoin de euh ben j'ai besoin de savoir si j'ai été claire et je ne le suis pas toujours hein c'est pas évident de + toujours bien formuler en plus euh (respire) parfois on a un truc technique qui est très clair et puis parfois c'est pas si clair que ça euh ++ ouais $\uparrow$ voilà c'est à peu près ça ça va ? (E-Céc, P11)

Celle-ci est de nature systématique ("je vérifie toujours") et s'appuie sur des indices corporels ou mimo-faciaux bien identifiés fonctionnant comme un stimulus pour l'enseignante qui produit une réponse verbale de reformulation. Son activité et la progression de son discours sont tributaires des signes (de leur nature et de leur quantité) qu'elle perçoit chez les étudiants. De plus, cette "pratique du regard", lors de la distribution de consignes, s'inscrit dans un format que l'enseignante présente comme stabilisé ("ça va être toujours mon fonctionnement") et contribue à la continuité signifiante, interactionnelle et relationnelle avec son public. Les discours de verbalisation mettent bien en exergue la 
manière dont se négocient ici le sens en interaction : par la mobilisation successive ou simultanée de perceptions visuelles et de l'activité langagière. L'action enseignante est avant tout interprétative. Enfin, son auto-reformulation ("j'ai un regard je PORTE un regard ") peut être l'indice d'une prise de conscience subite de ce "savoir perceptuel et de sa centralité"' au sein de son action didactique et pédagogique.

Par ailleurs, si les enseignantes parlent de leur regard en direction des apprenants, elles semblent également bien conscientes du regard de ces derniers sur elles. Ainsi dans cet extrait :

et je me rends compte que je je pose la question de la consigne et hum et en même temps XX mes affaires pour récupérer ma fiche péda (rires) tout ça CA je pense que c'est un de mes défauts enfin de mes défauts je pense qu'on est on est il y a beaucoup d'enseignants comme moi on aime pas trop les les temps morts j'ai envie de dire LE SILENCE ne me pèse pas mais le temps mort c'est celui où c'est MOI qui me qui me re:: euh enfin qui m'organise qui organise mes papiers qui re-range mon bureau pour retrouver mes papiers ça j'aime pas parce que je trouve que d'abord c'est pas une bonne image et que et puis les étudiants ils attendent donc en fait euh j'ai LU souvent ce que je fais c'est que je VoIS sur le document que je leur ai distribué euh la consigne ou les exercices à faire je POSE la consigne et ensuite je vais rechercher dans mes papiers pour retrouver mes documents donc je me rends compte que c'est pas très : : : c'est pas très avenant en fait de ma part parce que pendant ce temps-là qu'ils cherchent et qu'ils me répondent moi je suis en train de regarder le bureau alors moi j'essaye de les regarder quand même pour leur montrer que je les écoute mais c'est je trouve que ça donne pas l'image d'un enseignant qui est disponible à cent pour cent pour la réponse qu'ils vont m'apporter donc à mon avis ça il faudrait que j'y remédie remédie pardon (E-Vic, P12).

La visualisation après-coup de son action lui rend à la conscience que, si les apprenants sont sous son regard, elle ne peut échapper à celui des apprenants. Lorsque l'enseignant relâche l'attention visuelle et langagière sur les apprenants, il se justifie auprès du chercheur et catégorise ce court instant de "temps mort", alors que c'est un temps pour lui-même, ("re-range mes papiers pour retrouver mes papiers" ; "XXX mes affaires") et qualifie son comportement de "pas avenant", véhiculant "une image d'enseignant pas disponible à $100 \% "$. Elle rend explicite la nécessité ou l'obligation d'une présence ou d'une attention aiguë à l'autre, à la classe. Il lui faut exister sans discontinuer sous le regard de l'apprenant sous peine d'incompétence, ou au moins sous peine d'être en défaut avec une conception affichée de l'enseignement. Face au chercheur, l'observation et la verbalisation de sa conduite va à l'encontre d'une certaine représentation d'elle-même et de ses conduites professionnelles qu'elle souhaiterait véhiculer. Mais elle montre aussi que l'exercice de son action visuelle répond à des normes professionnelles concernant l'attention ("c'est pas très avenant en fait de ma part parce que pendant ce temps-là qu'ils cherchent et qu'ils me répondent moi je suis en train de regarder le bureau") et l'interaction, mais aussi le public d'apprenants ("avec un groupe A1 je ne vais pas parler euh quand je suis face au tableau je vais me retourner pour qu'ils me voient pour que ils lisent sur les lèvres un petit peu comme des malentendants"). Elle évoque également une norme attentionnelle et interactionnelle attachée au statut de l'apprenant :

ils sont déjà lancés dans la partie 1 et j'ajoute d'autres informations pour la partie 2 en fait bon je sais pas si à tort mais souvent je pense donc à tort on pense que les étudiants qui ne nous regardent pas ne nous écoutent pas et ne 
nous comprennent pas mais : : BON à mon avis ils peuvent faire deux choses en à la fois en même temps et si ça se trouve ils comprennent tout et ils vont faire ce que je leur ai demandé et a plupart du temps c'est ce qui se vérifie (EVic, P33) lequel si les étudiants ne regardent pas c'est qu'ils n'écoutent pas, pour mieux en prendre le contre-pied face au chercheur. Cette norme sur l'activité mono-attentionnelle attendue permet de justifier qu'elle continue la distribution de consigne alors que les apprenants sont déjà engagés dans la tâche. De son point de vue, cette norme est acceptable pour les apprenants (ils peuvent écouter même s'ils ne me regardent pas) mais pas pour ellemême (c'est pas avenant de ne pas les regarder alors qu'ils sont en train de me répondre). L'asymétrie interactionnelle n'est finalement peut-être pas toujours du côté que l'on croit.

\section{Discussion : de l'action visuelle en classe à la formation à une "vision professionnelle" ?}

"Voir" et "faire voir" semblent deux modes d'intervention tout à fait importants pour l'enseignant dans cette phase de distribution de consignes et de mise au travail des apprenants. La perception visuelle contribue également à façonner l'agir enseignant car elle constitue un médium fécond d'accumulation de l'expérience. Le dispositif réflexif instauré, alliant visionnage vidéo (lecture d'une action) et élaboration d'un discours (production d'une interprétation de cette action), peut amplifier cette focalisation sur le rôle de l'action visuelle. La vue est privilégiée, l'enseignant voit la classe, se voit et doit en produire une interprétation pour le chercheur. Cela peut avoir pour effet, dans le dispositif d'auto-confrontation, une "fragmentation dynamique de la perception" visuelle (Filliettaz, 2007: 21) et une accentuation des commentaires sur cette dimension perceptive. La fragmentation, décomposition de plus en plus fine de l'activité, est ainsi définie par Cahour (2006:6)

[Elle] permet de rendre explicite ce qui restait implicite dans la description de l'expérience vécue, soit parce qu'un acte cognitif particulier est nécessaire pour réfléchir le vécu qui est pré-réfléchi, inscrit dans l'action, et non disponible de façon immédiate pour la verbalisation, soit parce que le sujet pense que ce n'est pas intéressant à dire, soit parce qu'il préfère ne pas en parler, par souci de protection des faces. [...] La fragmentation est possible pour les actions (quand tu faisais cela, tu faisais quoi ?) mais aussi pour les activités perceptives (quand tu voyais cela, tu le voyais comment?), les sensations corporelles (quand tu te sentais inconfortable à ce moment-là, c'était comment ?), etc.

La référence déictique à l'action visionnée ("là", "ici", "je le vois tout de suite"), largement observable dans les discours d'auto-confrontation, est à ce titre un levier discursif de cette fragmentation. Elle permet ainsi d'accéder à certains ingrédients de l'action (la fabrication constante du sens), à des micro-finalités portées par l'enseignant et très liées aux situations qu'il rencontre, au degré d'attention, etc.

Cette fragmentation interroge finalement le rôle du regard dans la compétence professionnelle. À ce sujet, les travaux de Goodwin (1994) nous apportent quelques éclairages. Selon lui, les pratiques (discursives) des membres d'une profession observées dans le cours de leur activité professionnelle concourent à déterminer une vision 
professionnelle, à développer une manière de voir et de comprendre des événements, des phénomènes propres à leur profession. Il prend pour exemple les manières de regarder (" practices of seeing") des archéologues. Elles se construisent, dans l'échange et par l'observation et la classification de phénomènes (la couleur de la terre) ainsi que, par exemple, par l'utilisation négociée d'un nuancier (ayant été lui-même conçu historiquement à partir de cette activité intense d'observation des sols), afin de déterminer la couleur observée. Ils interprètent ainsi un phénomène et construisent des manières de voir socialement organisées. L'intérêt du concept est, selon nous, qu'il prend appui sur une mobilisation conjointe du discours en interaction et de l'action située des professionnels. Et il parait particulièrement fécond pour penser les dispositifs de formation basés sur le visionnage et le discours qui visent à produire et à confronter des visions professionnelles sur un phénomène didactique ou pédagogique par le croisement des interactions de classe et des discours tenus par l'enseignant sur celles-ci.

Les analyses réflexives pourraient être orientées par différents axes. Par exemple, l'extrait étudié dans le point 3.2.2 ci-dessus, montrant le rôle du retrait de la scène verbale ou d'une certaine prise de recul par rapport à la consigne distribuée, nous paraîtrait particulièrement fécond à travailler en formation autour du "regard évaluatif" de l'enseignant.

Dans un premier temps, il s'agirait de faire émerger les interprétations (pourquoi et que regarde l'enseignant dans les différentes phases didactiques?) et à répertorier les fonctions (voir pour voir s'ils sont au travail, voir pour voir s'il ne manque rien, etc.). Dans un deuxième temps, il conviendrait d'analyser le discours produit en autoconfrontation. D'abord, une analyse discursive pourrait être réalisée en recensant par exemple les formules du type "je me rends compte", "je vois", etc. ainsi que les déictiques, facilitant alors le repérage des indices pertinents de l'action (l'activité de "coding" pour Goodwin) susceptible de structurer progressivement le regard. Ensuite, suivrait une étape d'analyse des dires (que dit l'enseignant sur son regard?, quels sont les apports de son discours?, que découvrons-nous ?) permettant d'identifier des stratégies concernant la distribution de documents, des habitudes dans la gestion de consignes, etc.

Ces différentes étapes peuvent contribuer à une meilleure connaissance des savoirs d'actions des enseignants en matière de prescription. Elles conduiraient à :

- éclairer la multiplicité et la simultanéité d'événements, de finalités, de savoirs d'appui et de modalités à un instant $\mathrm{T}$.

- expliciter la manière de gérer la simultanéité des actions et des discours et par la même la simultanéité des foyers d'attention.

- faciliter une prise de conscience du rôle du regard comme ressource exploitable.

Il s'agit donc d'entraîner la pratique du regard comme mode d'élaboration de connaissances sur l'action et sur la classe, de développer des compétences d'observation et d'interprétation in vitro susceptibles d'être transférées in vivo, puisqu'

une composante importante de la compétence à enseigner est la capacité d'observer et d'interpréter les événements en classe lors d'une leçon et prendre des décisions d'enseignement basées sur ces interprétations (Borko et al., 2011 : 185, dans Gaudin \& Flandin, $2014: 40$ ). 


\section{Conclusion}

51 Nous avons tenté dans cette contribution de mettre en valeur certains aspects de l'activité prescriptive de l'enseignant. Premièrement, nous avons montré son caractère polyfocalisé : des actions de différente nature et des discours sont à organiser, des foyers d'attention parfois multiples sont à maîtriser. Cette polyfocalisation peut être source de tensions ou au moins nécessite, de la part de l'enseignant, une prise de décision et une lecture toujours renouvelée de ce qui advient dans la classe. De ce fait, les discours réflexifs donnent à voir en partie ce qui la sous-tend : des habitudes, la connaissance du public, le temps de la "respiration", etc.

Nous avons aussi centré notre analyse sur le rôle de la perception visuelle dans la prescription pour les enseignantes. Un triple regard a été posé sur elle : comment organiser efficacement l'exposition visuelle aux savoirs, le regard comme ressource de l'activité prescriptives et les normes attentionnelles attachées au statut de chacun dans la classe.

Finalement, les enseignants-informateurs se découvrent en tant que "prescripteurs" car

on ne peut être à la fois dans l'action et dans l'interaction avec les élèves ni tout comprendre de ce qui se passe: le temps de la compréhension vient souvent en différé, après le temps de l'action. C'est d'autant plus vrai lorsqu'il s'agit de situations complexes. (Vinatier, $2013: 47$ ).

Et l'étude des interactions de classe associée à celle des discours réflexifs, constitue un mode de lecture privilégié de l'action, que ce soit dans le cadre d'une recherche ou d'une formation. Il resterait toutefois à discuter plus amplement du rapport entre discours réflexifs pour la recherche et discours réflexifs pour la formation. Cela pourra faire l'objet d'une publication ultérieure.

\section{BIBLIOGRAPHIE}

Borko, H. et al. (2011). "Using video representations of teaching in practice-based professional development programs". ZDM Mathematics Education, vol. 43, n 1. pp. 175-187.

Cahour, B. (2006). "L'expérience vécue des utilisateurs : pourquoi l'étudier et comment ?" Actes du colloque CITE 2006 (Coopération, Innovation, Technologie), Université de Nantes.

Cicurel, F. (2011). Les interactions dans l'enseignement des langues. Agir professoral et pratiques de classe. Paris : Didier.

Filliettaz, L. (2002). La parole en action. Éléments de pragmatique psycho-sociale. Québec : Editions Nota bene.

Filliettaz, L. (2007). "On peut toucher ?" L'orchestration de la perception sensorielle dans des interactions en formation professionnelle initiale. Bulletin suisse de linguistique appliquée, $\mathrm{n}^{\circ} 85$. pp. 11-32. 
Gaudin, C. \& Flandin, S. (2014). "La vidéoformation dans tous ses états. Présentation croisée de l'état de l'art". Conférence de Consensus Janvier 2014. Non Publié.

Goffman, E. (1987). Façons de parler. Paris : Minuit.

Goodwin, C. (1994). "Professional vision". American Anthropologist n 96. pp. 606-633.

Goodwin, C. (2000). "Practices of Seeing : Visual Analysis. An Ethnomethodological Approach". In van Leeuwen, T. \& Jewitt, C. (dir.) Handbook of Visual Analysis. London : Sage Publications.

pp. 157-182

Grize, J.-B. (1996). Logique naturelle et communication. Paris : PUF.

Kendon, A. (1967). "Some functions of gaze direction in social interaction". Acta Psychologica, 26. pp. 22-47.

Mounin, G. ([1974]1993). Dictionnaire de la linguistique, PUF : Paris

Perrenoud, P. (2001). Développer la pratique réflexive dans le métier d'enseignant. Professionnalisation et raison pédagogique. Paris : ESF.

Sherin, M. G. \& Van Es, E. A. (2009). "Effects of vidéo club participation on teacher's professional vision". Journal of Teacher Education. $\mathrm{n}^{\circ}$ 60(1). pp. 20-37

Vinatier, I. (2013). La didactique professionnelle et le travail de l'enseignant. Bruxelles : De Boeck.

\section{NOTES}

1. Les termes "observateur de lui-même" et "position d'expertise" sont en italique dans le texte.

2. Corpus recueilli dans le cadre d'un projet de recherche mené par l'équipe Idap, autour de F. Cicurel en 2004.

3. Conventions de transcription communes aux deux corpus : + = pause ;: : = allongement; $\mathrm{X}=$ segment incompréhensible ; (rires) : aspect non verbal ou commentaire du transcripteur ; je SAIS : accentuation ; ?: intonation montante, interrogation ; Am : apprenant masculin ; Af : apprenant féminin ; As : plusieurs apprenants s'expriment ; oui : chevauchement ; (..) : segment coupé Corpus $1: \mathrm{CSML}=$ l'interaction-cours; $\mathrm{CCML}=$ commentaires enregistrés après chaque séance de cours $; \mathrm{ML}=$ Marie-Laure., $\mathrm{X}$ (stag) = co-stagiaire de ML

Corpus $2: \mathrm{E}-\mathrm{Isa}, \mathrm{P} 12$ = Entretien avec Isabelle, passage 21 de l'interaction-cours visionné ; $\uparrow$ ou $\downarrow$ : intonation montante ou descendante.

4. Nous avons volontairement supprimé dans cet extrait les segments de parole chevauchée (soulignées) afin de mettre en évidence (par soulignement) les conjonctions et relations temporelles entre ses actions.

5. "Un signe est dit motivé lorsque les raisons du choix de la forme sont transparentes" (Mounin, 1993 : 223).

6. Filliettaz (2007 : 15) parle de la "centralité des "savoirs perceptuels" dans la construction de certaines compétences professionnelles". 


\section{RÉSUMÉS}

L'activité de consigne est une activité majeure du processus d'enseignement et d'apprentissage. Ses enjeux sont bien connus: clarté informationnelle, compréhension des attentes de l'enseignant, autonomie, etc. Elle constitue également un puissant levier de la réalisation du projet de l'enseignant et de la mise au travail des élèves. Néanmoins, la dimension cachée de l'activité de consigne (les préoccupations de l'enseignant, les normes professionnelles, les intentions, les motifs présidant à l'élaboration et la distribution des consignes, les microdécisions) demeure encore peu renseignée. C'est, par conséquent, dans cette perspective que nous abordons l'activité de prescription. Nous nous intéressons à la dimension polyfocalisée de l'activité de consigne et de mise au travail des apprenants. Puis, nous mettons l'accent sur une modalité de gestion de la multi-activité : l'activité visuelle de l'enseignant associée à la passation des consignes. Le regard, comme activité attentionnelle, constitue un mode d'organisation et de lecture normé des événements sur lesquels il doit réagir in situ. Enfin, nous mettons en relation la situation d'auto-confrontation alliant visionnage de l'action et discours réflexifs avec la situation de classe où l'enseignant est aussi un observateur au regard aiguisé. Nous esquisserons ainsi les contours d'une "vision professionnelle" partagée et intéressante à mobiliser au regard des enjeux de formation des enseignants.

The instructional process is a vital component of teaching and learning. Its benefits are well known: it can provide clarity of information, comprehension of teacher expectations, autonomy, etc. In addition, it is has a great impact on student work and project completion. Nonetheless, the hidden dimension of the instructional process (instructor's concerns, professional norms, intentions, instructional design, micro-decisions) remains elusive. It is within this context that we approach the prescriptive instructional process. Our study will explore a multi-facetted focus for both the instructional process and student work. We will stress a specific approach to the management of this multi-layered activity: the visual component of the teacher's instructional process. Observation, as a reflective process, represents a learned comprehension of events to which the teacher is expected to react within and based upon the given context. Finally, we will contextualise the self-assessment process wherein teachers observe their own practice and proceed via reflective discourse within the classroom context in which the teacher is also an informed observer. We will thus outline the foundation of a shared and interesting "professional vision" within the context of instructor development.

\section{INDEX}

Keywords : teaching, instructions, multi-layered activity, observation, reflective discourses, professional vision

Mots-clés : agir enseignant, consignes, polyfocalisation, regard, discours réflexifs, vision professionnelle 


\section{AUTEURS}

\section{ESTEFANIA DOMINGUEZ}

Université Paris 3 - Sorbonne Nouvelle, France

Estefania Dominguez est enseignante contractuelle à l'université d'Angers, doctorante à

l'université Paris 3 et membre du laboratoire Diltec. Ses travaux portent sur l'agir multimodal de enseignants de FLE et sur les pratiques réflexives liées à la professionnalisation.

Courriel : estefania.dominguez[at]gmail.com

\section{VÉRONIQUE RIVIÈRE}

Université Lyon 2, France

Véronique Rivièe est maître de conférences à l'université Lumière Lyon 2 et membre du laboratoire Icar (UMR Cnrs). Ses travaux portent sur les pratiques professionnelles des enseignants de Fles et sur le interactions de type didactique

Courriel : veronique-riviere[at]univ-lyon2.fr 